\title{
Hämophilie-Patienten brauchen mehr als nur Gerinnungsfaktoren
}

\begin{abstract}
- Hämophilie ist eine seltene genetische Erkrankung, an der in Deutschland ca. 6000 Patienten leiden. Durch einen Mangel oder das Fehlen des Gerinnungsfaktors VIII (Hämophilie A) oder IX (Hämophilie B) kommt es immer wieder zu Blutungen in Muskeln, Gelenken und anderen Geweben. Das kann zu Langzeitschäden, insbesondere Gelenkversteifungen führen. „Da die Erkrankung nicht heilbar ist, muss der fehlende Gerinnungsfaktor mit den entsprechenden Faktorkonzentraten substituiert werden", sagte Dr. Katharina Holstein, Hamburg-Eppendorf. Die Substitution erfolgt im Allgemeinen prophylaktisch, wobei der fehlende Gerinnungsfaktor zweibis dreimal pro Woche intravenös injiziert wird. Damit hätten Betroffene heute eine normale Lebenserwartung.
\end{abstract}

„Die Hämophilie-Behandlung ist jedoch mehr als nur die Verordnung und Injektion des Gerinnungsfaktors", so Holstein. Der Alltag ist geprägt von vielfältigen Herausforderungen, v. a. psychosozialen Problemen, die ein Leben mit Hämophilie für die Betroffenen selbst und deren Familie schwer machen könnten.

Mit dem Bayer-Förderpreis PHILOS sollen keine wissenschaftlichen Forschungsarbeiten, sondern vorbildliche Betreuungskonzepte und besonderes soziales Engagement für Hämophilie-Patienten gewürdigt werden. „Dies können Schulungs- und Freizeitprojekte für Kinder, Betreuungsangebote speziell für ältere Patienten, Möglichkeiten zum Erfahrungsaustausch oder zur Entlastung der betroffenen Familien sein", so Dr. Klaus Horn, Geschäftsleiter Hämosta-

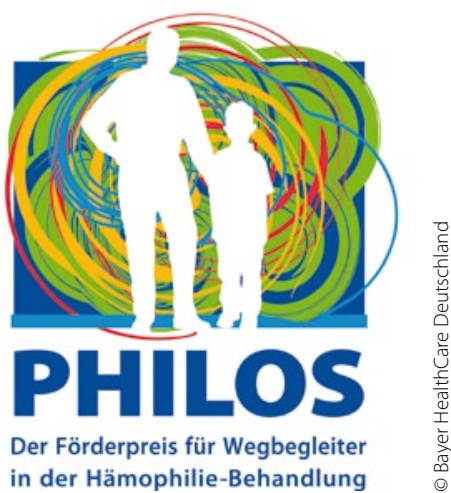

seologie bei Bayer HealthCare Deutschland. Der erste Platz ist mit 10000, der zweite mit 5000 und der dritte mit 2500 Euro dotiert. Bis 30. September 2012 können sich Ärzte, Hämophilie-Schwestern, Physio- und Psychotherapeuten wie auch Selbsthilfegruppen und Vereine bewerben. Weitere Informationen unter: www.philos.bayer.de.

- Dr. med. Peter Stiefelhagen

Quelle: PHILOS Presse-Vorstellung, Jahrestagung der GTH, St. Gallen, Februar 2012 (Veranstalter: Bayer HealthCare Deutschland)

\section{Gastroösophagealer Reflux \\ Sodbrennen ist nicht gleich Sodbrennen}

— Klagen Patienten über einen sauren Magen oder Aufstoßen, haben sie noch lange keine Refluxerkrankung, sondern häufig eine dyspeptische Magenstörung, bei der eine andere Therapie nötig ist. Damit dieser Tatsache mehr Aufmerksamkeit geschenkt wird, auch von Ärzten und Apothekern, startet eine bundesweite Aufklärungskampagne zum Thema Sodbrennen, die von Gastroenterologen und Apothekern des Kompetenzteams Magen, der Deutschen Gesundheitshilfe und dem Unternehmen Steigerwald unterstützt wird.

\section{Infomobil tourt durch Deutschland}

Herzstück der Kampagne ist ein Infomobil, das beim Internistenkongress startet und bis 20. Juni durch 48 Städte tourt. Ein Arzt berät Interessierte, es gibt Anamnese-Fragebögen, Aufklärungsmaterialien, Ernährungstipps (u.a. magenfreundliche Rezepte von Sterne-Köchen), Patientenbroschüren, Schnelltests und Plakate fürs Wartezimmer.
„Sodbrennen wird von vielen Patienten lange Zeit hingenommen. Sie gehen davon aus, selbst schuld zu sein, etwa, weil sie etwas Falsches gegessen haben", erklärte Prof. Peter Malfertheiner, Magdeburg. Gingen sie dann zum Arzt, bekämen sie fast selbstverständlich einen Protonenpumpenhemmer (PPI) verordnet. „Die Einführung der Protonenpumpenhemmer hat zweifellos einen Meilenstein in der Behandlung der Refluxösophagitis gesetzt. Aber es hat sich damit auch eine Routine eingeschlichen, sodass das Symptom Sodbrennen oft zu wenig differenziert betrachtet wird," warnte der Gastroenterologe.

Tatsächlich habe nur etwa ein Drittel der Patienten mit Sodbrennen eine erosive Refluxkrankheit. Bei zwei Dritteln zeige sich trotz Beschwerden ein endoskopisch normales Bild, ergänzte Prof. Hans-Dieter Allescher, Garmisch-Partenkirchen. Hier seien PPI nicht indiziert.
Malfertheiner forderte, bei der Anamnese weitere Symptomen (ROM-III-Kriterien) zu erfragen. Probatorisch könnten bei starken Refluxbeschwerden PPI für zwei Wochen verordnet werden. Bei anhaltender Symptomatik sei jedoch eine weitergehende Diagnostik einschließlich Endoskopie unbedingt nötig. Spreche der Befund für eine funktionelle Dyspepsie, sind nach seiner Erfahrung Phytotherapeutika eine effektive und verträgliche Option - auch bei Sodbrennen. Dies bestätigte eine kontrollierte Untersuchung mit Iberogast ${ }^{\circledR}$, in der mit dem Extrakt STW 5 nach 14 Tagen eine signifikant stärkere Symptomlinderung nach dem Gastrointestinal Symptom Score (GIS) erreicht wurde als mit Placebo (Am J Gastroenterol. 2007;102(6):1268-75).

- Ruth Ney

Quelle: Pressekonferenz „Initiative Gesunder Magen, Sodbrennen - ein Symptom, zwei häufige Krankheitsbilder", München, Februar 2012 (Veranstalter: Steigerwald) 